[0212-7199 (2003) 20: 6; pp 312-316] ANALES DE MEDICINA INTERNA Copyright $\odot 2003$ ARAN EDICIONES, S.L.

An. MEd INTERNa (Madrid) Vol. 20, N. $^{\circ} 6$, pp. 312-316, 2003

\title{
Endocarditis por Stenotrophomonas maltophilia: presentación de un caso y revisión de la literatura
}

\author{
R. LÓPEZ RODRÍGUEZ, F. L. LADO LADO, I. RODRÍGUEZ LÓPEZ, R. GAMALLO \\ THEODOSIO, V. LORENZO ZÚÑIGA, L. RODRÍGUEZ-OTERO' \\ Servicios de Medicina Interna y de 'Microbiología. Departamento de Medicina. Complejo \\ Hospitalario Universitario de Santiago de Compostela. Santiago de Compostela. A \\ Coruña
}

\begin{abstract}
ENDOCARDITIS CAUSED BY STENOTROPHOMONAS MALTOPHILIA: REPORT OF A CASE AND REVIEW OF LITERATURE
\end{abstract}

\section{RESUMEN}

Se presenta el caso de una mujer de 65 años con una endocarditis tardía localizada en la válvula protésica aórtica y absceso paravalvular producida por Stenotrophomonas maltophilia. El proceso debuta, de forma aguda con la rotura del referido absceso que cursa con fiebre, bacteriemia e insuficiencia cardíaca congestiva secundaria a insuficiencia aórtica severa. La identificación de Stenotrophomonas maltophilia, como agente responsable, representa una excepcional causa de endocarditis pues sólo 22 casos han sido descritos en la literatura médica y la mayoría de ellos en adictos a drogas por vía parenteral o como complicación del recambio valvular cardíaco. Hemos realizado una revisión sobre las características epidemiológicas, clínicas y pronósticas de esta enfermedad, así como de su tratamiento y prevención.

PALABRAS CLAVE: Stenotrophomonas maltophilia. Xanthomonas maltophilia. Pseudomonas maltophilia. Endocarditis.

\begin{abstract}
We report a case of a 65 year-old woman with late endocarditis of prosthetic aortic valve and paravalvular abscess by Stenotrophomonas maltophilia, which had an acute presentation for the memtionated abscess broken, with fever, bacteremia and congestive heart failure secondary to severity aortic regurgitation. It's a rare cause of endocarditis with only 22 cases descripted in medical literature, the most of them in parenteral drug addict and as complication of cardiac valve replacement. The literature is reviewed and relate the epidemiology, clinical and prognosis characteristics of this disease, the same as his treatment and prevention.
\end{abstract}

KEY WORDS: Stenotrophomonas maltophilia. Xanthomonas maltophilia. Pseudomonas maltophilia. Endocarditis.

López Rodríguez R, Lado Lado FL, Rodríguez López I, Gamallo Theodosio R, Lorenzo Zúñiga V, Rodríguez-Otero L. Endocarditis por Stenotrophomonas maltophilia: presentación de un caso y revisión de la literatura. An Med Interna (Madrid) 2003; 20: 312-316.

\section{INTRODUCCIÓN}

La Xanthomonas maltophilia (1), conocida hasta 1983 como Pseudomonas maltophilia (2), es una bacteria gramnegativa, no fermentadora, aerobia y móvil mediante varios flagelos polares, que es reclasificada como el único miembro del género Stenotrophomonas en 1993 (3). La S. maltophilia es un microorganismo ubicuo que ha sido aislado en numerosas fuentes de agua: ríos, pozos, lagos, agua embotellada, y aguas negras; suelo; animales; alimentos; plantas y residuos orgáni$\cos (4-10)$.

En la última década, ha emergido como una importante causa de infección nosocomial, en particular en individuos inmunodeprimidos o con enfermedades crónicas debilitantes. Sus principales factores de virulencia incluyen la resistencia a múltiples antimicrobianos, la adherencia a materiales plásticos y la producción de exoenzimas como la elastasa y la gelatinasa. Esta bacteria constituye una extraña causa de endocarditis (11-27).

A continuación, exponemos el caso de una paciente con endocarditis de válvula aórtica protésica y absceso paravalvular. La sintomatología se muestra de forma aguda con relación a la rotura del citado absceso, presentando fiebre, bacteriemia e insuficiencia cardíaca congestiva secundaria a

\footnotetext{
Trabajo aceptado: 16 de octubre de 2002
} 
insuficiencia aórtica severa. Hemos efectuado una revisión de las características epidemiológicas, clínicas y pronósticas de esta enfermedad, así como de su tratamiento y prevención. Para ello, se realizó una búsqueda, en MEDLINE y en BIOMEDNET, de estudios relacionados con el caso clínico aportado, utilizando como palabras clave: Stenotrophomonas maltophilia, Xanthomonas maltophilia, Pseudomonas maltophilia y endocarditis. Hallamos 22 casos descritos de endocarditis por S. maltophilia, de los que se recopilaron los datos proporcionados acerca de edad de los pacientes, tipo de infección (precoz o tardía), válvulas afectadas (nativas o protésicas), factores de riesgo, aislamiento del microorganismo, tratamiento empleado (antibióticos y/o cirugía), complicaciones y pronóstico (Tabla I).

\section{CASO APORTADO}

Se trata de una mujer de 65 años de edad, con antecedentes de recambio valvular aórtico (prótesis mecánica Carbomedics $21 \mathrm{~mm}$ ) por doble lesión aórtica en noviembre de 2000. Al cabo de tres meses (febrero de 2001), la paciente ingresa por una celulitis en la extremidad inferior izquierda, que evolucionó favorablemente tras el tratamiento con cloxacilina intravenosa. Los hemocultivos resultaron negativos y un ecocardiograma transesofágico objetivó una prótesis aórtica normofuncionante.

Una vez transcrurridos cuatro meses se decide, nuevamente, su ingreso hospitalario por presentar un cuadro clínico de astenia, anorexia y pérdida de peso con evidencia de una anemia microcítica. No había antecedentes de sangrado digestivo, ginecológico ni de alteraciones del hábito intestinal. En la exploración física destacaba palidez cutáneo-mucosa y auscultación de un soplo sistólico en foco aórtico. En la analítica realizada a su ingreso se hallaban, además de la mencionada anemia, los siguientes valores: VSG de $103 \mathrm{~mm}$ a la primera hora, urea $96 \mathrm{mg} / \mathrm{dl}$, creatinina $2,9 \mathrm{mg} / \mathrm{dl}$ y albúmina de 2,9 $\mathrm{g} / \mathrm{dl}$. La fórmula leucocitaria, el resto de los parámetros bioquímicos y la analítica elemental de orina fueron normales. El electrocardiograma y la radiografía de tórax no mostraron alteraciones de relevancia.

A los pocos días de su ingreso la paciente tuvo fiebre $\left(39,5^{\circ} \mathrm{C}\right)$ y disnea. En la exploración física se observó taquipnea, taquicardia, ingurgitación yugular, soplo de doble componente en foco aórtico, crepitantes diseminados en ambos campos pulmonares y edemas con fóvea en ambas extremidades inferiores. En cuanto a las pruebas complementarias, en la gasometría arterial había alcalosis e insuficiencia respiratoria $\left(\mathrm{pO}_{2} 40 \mathrm{mmHg}\right)$ y en el control analítico se apreció -además de la anemia y los datos bioquímicos de insuficiencia renal referidos- leucocitosis $(13.930 / \mu \mathrm{L})$ con neutrofilia (neutrófilos $83 \%$ ) y desviación izquierda (Cayados 7\%). En la radiografía de tórax se evidenciaron signos de enfermedad difusa bilateral mixta y derrame pleural derecho. En el ecocardiograma transtorácico se contempló disfunción de la prótesis aórtica con insuficiencia aórtica de moderada a severa; en el ecocardiograma transesofágico se pudo percibir prótesis aórtica con dehiscencia del anillo de aproximadamente un tercio de su circunferencia con imágenes móviles que ocasiona insuficiencia aórtica severa. Con los resultados obtenidos, la paciente fue sometida a cirugía cardiaca urgente con sustitución valvular, sutura del absceso y extirpación de la vegetación localizada en la intercomisura derecha-izquierda.

Los hemocultivos recogidos durante el episodio agudo, los cultivos de la vegetación, la válvula protésica y en el pus del absceso fueron positivos para Stenotrophomonas maltophilia. Se instauró tratamiento antimicrobiano -según antibiograma- con ceftazidima y gentamicina, a dosis ajusatadas según la función renal, con buena respuesta clínica.

\section{DISCUSIÓN}

La Stenotrophomonas maltophilia, después de la $P$. aeruginosa, representa el bacilo gramnegativo que con más frecuencia se aísla en especímenes clínicos (28-30). Aunque es un patógeno preferentemente nosocomial, en los últimos años se ha puesto de manifiesto que la infección adquirida en la comunidad es más habitual de lo que, en un principio, se pensaba (31-33). Se reconocen como fuentes potenciales de la infección hospitalaria los siguientes factores: agua destilada, agua de los servicios, nebulizadores, catéteres, esfingomanómetros, termómetros, soluciones desinfectantes, máquinas de diálisis, fluidos intravenosos, ventiladores, máquinas de hielo y las manos del personal sanitario (34-39).

La S. maltophilia ha sido identificada como agente responsable de una amplia variedad de procesos infecciosos tales como la neumonía, la bacteriemia, las infecciones del tracto urinario, meningitis, la infección de la herida quirúrgica, mastoiditis, epididimitis, endocarditis y celulitis (40). Los factores de riesgo más usuales en la adquisición de la infección son los relacionados con una terapia antimicrobiana previa, catéter venoso central, hospitalización prolongada, estancia en UCI, ventilación mecánica, neutropenia, neoplasias, tratamiento citostático, corticoides y exposición a pacientes infectados (31,37,41-44).

Este germen supone una singular y grave causa de la endocarditis, con sólo 23 casos, incluido el que exponemos, descritos en la literatura médica (11-27). La mayoría se produjeron en pacientes con válvulas cardíacas protésicas $(60 \%)$, siendo la más comúnmente afectada la aórtica (8/14). Su forma de presentación resultó precoz (dentro de los dos primeros meses postcirugía), en tan sólo 5 casos (36\%), mientras que en los 9 restantes (64\%) la aparición fue tardía (1116,19,20,24,26,27), es decir, la infección surgió transcurridos 2 ó más meses del postoperatorio, y a este grupo pertenece nuestro caso. En más del $50 \%$ de los episodios, se identificaron los factores de riesgo que a continuación mencionamos: adicción a drogas por vía parenteral, cirugía cardíaca, tratamiento dental, shunt ventrículo-atrial, cistoscopia sin profilaxis y catéteres venosos centrales $(12,15,17-19,21-23,25)$.

En el presente caso, valoramos la posibilidad de que la paciente contrajera la infección en su ingreso previo por una celulitis en la extremidad inferior izquierda y que, posteriormente, desarrollara un absceso paravalvular que bién podría ser al romperse, el responsable del cuadro de bacteriemia e insuficiencia cardíaca congestiva. Sin embargo, esta hipótesis parece poco probable, en nuestro caso, porque el ecocardiograma transesofágico practicado, en dicho ingreso, resultó normal. Por tal motivo, pensamos que, aunque lo comentado no se puede descartar, sería más factible que se tratara de una infección adquirida en la comunidad.

En cuanto a las complicaciones originadas en el seno de esta infección, una vez examinados los casos descritos (1127), se concluye que las más frecuentes son la insuficiencia cardíaca congestiva, los émbolos sépticos, los abscesos paravalvulares o miocárdicos y la bacteriemia persistente.

Al abordar el apartado del tratamiento, en la mayoría de los casos (11-27), se utilizaron dos o más fármacos y se procedió a la intervención quirúrgica con recambio valvular en once de los pacientes. La S. maltophilia es resistente a múltiples agentes antimicrobianos, especialmente a los b-láctamicos, debido a que presenta, al menos, cuatro tipos de b-lacta- 
TABLA I

CARACTERÍSTICAS DE LOS 23 PACIENTES CO N ENDO CARDITIS PO R STENO TRO PHO M O NAS M ALTO PHILIA

\begin{tabular}{|c|c|c|c|c|c|c|c|c|c|}
\hline Caso & Año (ref.) & $\begin{array}{l}\text { Edad / } \\
\text { sexo }\end{array}$ & $\begin{array}{l}\text { Tipo de } \\
\text { infección }\end{array}$ & $\begin{array}{l}\text { Factores de } \\
\text { riesgo }\end{array}$ & Cultivo & Tratamiento & Cirugía & Complicación & Pronóstico \\
\hline 1 & 1967 (11) & $26 / V$ & Precoz VPM & Ninguno & Sangre & $\begin{array}{l}\text { Cloranfenicol, } \\
\text { kanamicina, } \\
\text { colistina }\end{array}$ & Sí & Émbolos sépticos & Exitus \\
\hline 2 & 1967 (11) & $30 / \mathrm{V}$ & Precoz VPM & Ninguno & & Cloranfenicol & No & Ninguna & Curación \\
\hline 3 & $1973(12)$ & $65 / M$ & Tardía VPM & Cistoscopia & Sangre, válvula & $\begin{array}{l}\text { Carbenicilina, } \\
\text { gentamicina, } \\
\text { kanamicina, } \\
\text { cloranfenicol, } \\
\text { polimixina B }\end{array}$ & Sí & $\begin{array}{l}\text { Bacteriemia } \\
\text { persistente }\end{array}$ & Curación \\
\hline 4 & $1973(13)$ & $35 / V$ & Precoz VPM & Ninguno & & $\begin{array}{l}\text { Gentamicina + } \\
\text { carbenicilina, } \\
\text { TM P-SM Z }\end{array}$ & No & Ninguna & Curación \\
\hline 5 & 1978 (14) & $38 / V$ & Tardía VPM & Ninguno & Sangre, válvula & $\begin{array}{l}\text { Estreptomicina + } \\
\text { penicilina }\end{array}$ & No & $\begin{array}{l}\text { Émbolos sépticos } \\
\text { IAM }\end{array}$ & Exitus \\
\hline 6 & $1978(15)$ & $22 / \mathrm{V}$ & Tardía VPA & ADVP & Sangre, válvula & $\begin{array}{l}\text { Amikacina + } \\
\text { carbenicilina + } \\
\text { TM P-SM Z }\end{array}$ & Sí & $\begin{array}{l}\text { Émbolos sépticos } \\
\text { ICC }\end{array}$ & Curación \\
\hline 7 & $1978(15)$ & $31 / V$ & Precoz VPA & $\begin{array}{l}\text { ADVP } \\
\text { Extracción } \\
\text { dental }\end{array}$ & Sangre, válvula & $\begin{array}{l}\text { Kanamicina + } \\
\text { carbenicilina } \\
\text { TM P-SMZ }\end{array}$ & Sí & $\begin{array}{l}\text { Absceso } \\
\text { perivalvular }\end{array}$ & Curación \\
\hline 8 & $1978(15)$ & $57 / \mathrm{V}$ & VNA,VNM & Ninguno & & $\begin{array}{l}\text { Polimixina B + } \\
\text { TM P-SM Z }\end{array}$ & No & Émbolos sépticos & Curación \\
\hline 9 & $1984(16)$ & $25 / \mathrm{V}$ & $\begin{array}{l}\text { Tardía postre- } \\
\text { paración DSV }\end{array}$ & Ninguno & Sangre, válvula & $\begin{array}{l}\text { Cloranfenicol, } \\
\text { gentamicina }\end{array}$ & No & Exitus & \\
\hline 10 & 1987 (17) & $33 / \mathrm{V}$ & VNA, VNT & ADVP & Sangre & $\begin{array}{l}\text { Ticarcilina } \\
\text { TM P-SM Z } \\
\text { M oxalactam }\end{array}$ & Sí & $\begin{array}{l}\text { ICC } \\
\text { Abscesos } \\
\text { miocárdicos }\end{array}$ & Exitus \\
\hline 11 & $1990(18)$ & NA & VNT & $\begin{array}{l}\text { CVC } \\
\text { infectado }\end{array}$ & & NA & NA & NA & Exitus \\
\hline 12 & $1990(18)$ & NA & VNA & Ninguno & NA & & NA & NA & Exitus \\
\hline 13 & 1990 (18) & NA & VNA & $\begin{array}{l}\text { CVC } \\
\text { infectado }\end{array}$ & NA & & NA & NA & Curación \\
\hline 14 & $1991(19)$ & $33 / V$ & Tardía VPA & $\begin{array}{l}\text { ADVP. Tto. } \\
\text { dental }\end{array}$ & & $\begin{array}{l}\text { TM P-SM Z + } \\
\text { ampicilina + } \\
\text { gentamicina }\end{array}$ & Sí & $\begin{array}{l}\text { Absceso } \\
\text { perivalvular }\end{array}$ & Curación \\
\hline 15 & $1992(20)$ & $56 / \mathrm{V}$ & Precoz VPA & Ninguno & Sangre, válvula & $\begin{array}{l}\text { Ceftazidima, } \\
\text { gentamicina, } \\
\text { TM P-SM Z }\end{array}$ & Sí & Émbolos sépticos & Exitus \\
\hline 16 & $1994(21)$ & $32 / \mathrm{V}$ & VNT & $\begin{array}{l}\text { ADVP, } \\
\text { reservorio SC }\end{array}$ & TM P-SM Z & No ICC & Exitus & & \\
\hline 17 & $1995(22)$ & $28 / \mathrm{V}$ & VNA & ADVP & & $\begin{array}{l}\text { Gentamicina + } \\
\text { ciprofloxacino }\end{array}$ & Sí & $\begin{array}{l}\text { Absceso } \\
\text { miocárdico }\end{array}$ & Curación \\
\hline 18 & $1996(23)$ & $60 / M$ & VNT & CVA & Sangre & $\begin{array}{l}\text { TM P-SM Z + } \\
\text { Ticarcilina-clav. }\end{array}$ & No & Absceso pulmonar & Curación \\
\hline 19 & $1998(24)$ & $\begin{array}{l}69 / M \\
\text { VPM }\end{array}$ & Tardía VPA, & Ninguno & Sangre, válvula & $\begin{array}{l}\text { Ceftazidima, } \\
\text { gentamicina, } \\
\text { ciprofloxacino, } \\
\text { TM P-SM Z }\end{array}$ & Sí & $\begin{array}{l}\text { ICC, bacteriemia } \\
\text { persistente }\end{array}$ & Exitus \\
\hline 20 & $1998(25)$ & $36 / V$ & VNA & Tto. dental & & $\begin{array}{l}\text { Piperacilina-taz. } \\
\text { ciprofloxacino }\end{array}$ & Sí & ICC & Curación \\
\hline 21 & $1998(26)$ & $40 / \mathrm{V}$ & Tardía VPA & Ninguno & Sangre & $\begin{array}{l}\text { Ticarcilina-clav, } \\
\text { +TMP-SM Z }\end{array}$ & No & Ninguna & Curación \\
\hline 22 & $2000(27)$ & $62 / \mathrm{V}$ & Tardía VPA & Ninguno & Sangre & $\begin{array}{l}\text { Cloranfenicol + } \\
\text { ciprofloxacino }\end{array}$ & No & Disección aórtica & Curación \\
\hline 23 & $\begin{array}{l}\text { Caso } \\
\text { actual }\end{array}$ & $65 / M$ & Tardía VPA & Ninguno & $\begin{array}{l}\text { Sangre, válvula } \\
\text { pus absceso }\end{array}$ & $\begin{array}{l}\text { Ceftazidima + } \\
\text { gentamicina }\end{array}$ & $\begin{array}{l}\text { Sí } \\
\text { a }\end{array}$ & $\begin{array}{l}\text { ICC, bacteriemia, } \\
\text { bsceso paravalvular }\end{array}$ & Curación \\
\hline
\end{tabular}

ADVP: Adicto a drogas por vía parentera; Clav.: clavulánico; CVA: cateter ventrículo-atrial; CVC: cateter venoso central; DSV: defecto septal ventricular; IAM : infarto agudo de miocardio; ICC: insuficiencia cardíaca congestiva; M: mujer; NA: no aportado; SC: subcutáneo; TAZ: tazobactam; TM PSM Z: trimetropim-sulfametoxazol; TTO : tratamiento; V: varón; VNA: válvula nativa aórtica; VNM : válvula nativa mitral; VNT: válvula nativa tricúspide; VPA: válvula protésica aórtica; VPM : válvula protésica mitral.PRECOZ: f 2 meses tras cirugía.TARDÍA: > 2 meses. 
masas (45). En buena parte de los estudios, el antibiótico de primera elección, para esta bacteria, es el trimetoprim-sulfametoxazol, a la máxima dosis tolerada (46-49). No obstante, parece ser más efectivo cuando se asocia a la ticarcilina-clavulánico (44). Así mismo, es de vital importancia, la retirada del material protésico y de los catéteres infectados $(41,44,50)$.

En nuestro caso, se realizó cirugía cardíaca urgente para la sustitución de la válvula y se instauró tratamiento antibiótico empírico con gentamicina y ceftazidima. Esta combinación se mantuvo, debido a que, por antibiograma, el germen resultó ser sensible a los citados fármacos. Pese a ello, una vez revisada la literatura, consideramos que el tratamiento antimicrobiano de elección para la endocarditis, de no disponer de la sensibilidad del microorganismo, debe ser la asociación de cotrimoxazol y ticarcilina-clavulánico. El recambio valvular estaría indicado, sobre todo, en la endocarditis protésica tardía, donde parece aumentar la supervivencia con respecto al manejo conservador ( $80 \%$ con cirugía y $50 \%$ sin ella) $(12,14-$ $16,19,24,26,27)$.

\section{Bibliografía}

1. Swings J, de Vos P, Van den Mooter M, de Ley J. Transfer of Pseudomonas maltophilia to the genus Xanthomonas as Xanthomonas maltophilia (Hugh 1981) comb. Nov. Int J Syst Bacteriol 1983; 33: 409-413.

2. Hugh R, Ryschenkow E. Pseudomonas maltophilia, an Alkaligenes-like species. J Gen Microbiol 1961; 26: 123-132.

3. Palleroni NJ, Bradbury JF. Stenotrophomonas, a new bacterial genus for Xanthomonas maltophilia (Hugh 1980) Swings et al. 1983. Int J Syst Bacteriol 1993;43:606-609.

4. Hugh R, Gilardi GL. Pseudomona. In: Lennette EH, Balows A, Hausler W Jr, Truant JP (ed.), Manual of clinical microbiology, 3rd ed. Washington, D.C.: American Society for Microbiology, 1980. p. 288-317.

5. Hugh R, Leifson E. A description of the type strain of Pseudomonas maltophilia. Int Bull Bacteriol Nomencl Taxon 1963; 13: 133-138

6. Hugh R, Ryschenkow E. An Alcaligenes-like Pseudomonas species. Bacteriol Proc, 1960. p. 78.

7. Guerzoni ME, Lanciotti R, Sinigaglia M, Gardini F. Análisis of the interaction between autochthonous bacteria and packaging material in PVC-bottled mineral water. Microb Res 1994; 149: 115-122.

8. Hernández-Duquino H, Rosenberg FA. Antibiotic- resistant Pseudomonas in bottled drinking water. Can J Microbiol 1987; 33: 286-289.

9. Kerr KG; Wilkinson FH. Bottled water as a source of Stenotrophomonas maltophilia and Pseudomonas spp for neutropenic patients. Eur J Cancer Care (in press).

10. Papapetropolou M, Iliopolou J, Rodopolou G, Detorakis J, Paniara O. Occurrence and antibiotic resistance of Pseudomonas species isolated from drinking water in southern Greece. J Chemother 1994; 6: 111-116.

11. Yeh TJ, Anabtawi IN, Cornett VE, White A, Stern WH, Ellison RG. Bacterial endocarditis following open-heart surgery. Ann Thorac Surg 1967; 3: 29-36.

12. Dismukes WE, Karchmer AW, Buckley MJ, Austen WG, Swartz MN Prosthetic valve endocarditis. Analysis of 38 cases. Circulation 1973; 48: 365-377.

13. Fischer JJ. Pseudomonas maltophilia endocarditis after replacement of mitral valve: a case study. J Infect Dis 1973; 128S: 771-773.

14. Semel JD, Trenholme GM, Harris AA, Jupa JE, Levin S. Pseudomonas maltophilia pseudosepticemia. Am J Med 1978; 64: 403-406.

15. Yu VL, Rumans LW, Wing EJ, McLeod R, Sattler FN, Harvey RM et al. Pseudomonas maltophilia causing heroin-associated infective endocarditis. Arch Inernt Med 1978; 138: 1667-1671.

16. Subbannayya K, Ramnarayan K, Shivananda PG, Shatapathy P, Mathai A. Pseudomonas maltophilia endocarditis. Ind J Pathol Microbiol 1984; 27: 311-315.

17. Muder RR, Yu VI, Dummer JS, Vinson C, Lumish RM. Infection caused by Pseudomonas maltophilia: expanding clinical spectrum. Arch
Las medidas que se pueden adoptar para prevenir la infección consisten tanto en evitar el uso inapropiado de antibióticos y la implantación prolongada de dispositivos externos (47), como en el mantenimiento y la desinfección o esterilización de los equipos de terapia respiratoria, hemodializadores y maquinas de hielo (34-38). Además, en los pacientes neutropénicos, se debe eludir el consumo de agua embotellada no carbonatada (9). También, como profilaxis y durante las epidemias nosocomiales, tiene especial importancia, la higiene de las manos y el uso de guantes por parte del personal sanitario $(31,37)$.

Como conclusión, es necesario tener presente que la Stenotrophomonas maltophilia, sin perjuicio de su baja incidencia, pueder ser el agente responable de una endocarditis, especialmente en pacientes portadores de una prótesis valvular. Con ello, se procura el establecimiento de un diagnóstico precoz, a la par que el uso inmediato de las medidas terapeúticas apropiadas para conseguir la reversibilidad del cuadro clínico, lo que evitaría consecuencias fatales.
Intern Med 1987; 147: 1672-1674.

18. Etling LS, Bodey GP. Septicemia due to Xanthomonas species and nonaeruginosa Pseudomonas species: increasing incidence of catheter related infections. Medicine (Baltimore) 1990; 69: 296-306.

19. Baddour LM, Meyer J, Henry B. Polymicrobial infective endocarditis in the 1980s. Rev Infect Dis 1991; 13: 963-970.

20. Jang TN, Wang FD, Wang LS, Liu CY. Xanthomonas maltophilia bacteremia: an analysis of 32 cases. J Form Med Assoc 1992 ; 92: 11701176.

21. Soriano V, Valencia E, Alba A, González-Lahoz J. Endocarditis por Xanthomonas maltophilia en un paciente con SIDA. Med Clin 1994; 102: 9.

22. Onate J, Aguirrebengoa K, Ibáñez de Maeztu JC, Hernández JL, Montejo M. Endocarditis aortica por Xanthomonas maltophilia en un paciente ADVP y revisión de la literatura. Enf Inf Microbiol Clin 1995; 13: 188189.

23. Rodero FG, Masia M, Cortes J, de la Tabla VO, Mainar V, Vilar A. Endocarditis caused by Stenotrophomonas maltophilia: case report and review. Clin Infect Dis 1996 ; 23: 1261-1265.

24. Munter RG, Yinnon AM, Schlesinger Y, Hershko C. Infective endocarditis due to Stenotrophomonas (Xanthomonas) maltophilia. Eur J Clin Microbiol Infect Dis 1998; 17: 353-356.

25. Shimoni S, Abend Y, Shimon A, Landau Z, Caspi A. Stenotrophomonas maltophilia endocarditis following dental treatment in a previously healthy patient. J Infect 1998; 37: 305-306.

26. Aydin K, Koksal I, Kaygusuz S, Kaklikkaya I, Caylan R, Ozdemir R. Endocarditis caused by Stenotrophomonas maltophilia. Scand J Infect Dis $2000 ; 32$ : 427-430.

27. Mehta NJ, Khan IA, Mehta RN, Gulati A. Stenotrophomonas maltophilia endocarditis of prosthetic aortic valve : recase and review of literature. Heart Lung 2000; 29: 351-355.

28. Goze A. Incidence des Pseudomonas sans pigment et des Moraxella en milieu hospitalier. Rev Epidemiol Med Sec Sante Publique 1973 ; 21: 499-520.

29. Holmes B, Lapage SP, Easterling BG. Distribution in clinical material and identification of Pseudomona maltophilia. J Clin Pathol. 1979; 32: 66-72.

30. Rosenthal SL. Sources of Pseudomonas and Acinetobacter species found in human culture. Am J Clin Pathol 1974; 62: 807-811.

31. Heath T, Currie B. Nosocomial and community acquired Xanthomonas maltophilia infection in tropical Australia. J Hosp Infect 1995; 30: 309313

32. McDonald GR, Pernenkil R. Community-acquired Xanthomonas maltophilia pyelonephritis. South Med 1993; 86: 967-968. 
33. Laing FPY, Ramotar K, Read RR, Alfieri N, Kureishi A, Henderson EA, Louie TJ. Molecular epidemiology of Xanthomonas maltophilia colonization and infection in the hospital environment. J Clin Microbiol 1995;33:513-518.

34. Fisher MC, Long SS, Roberts EM, Dunn JM, Balsara RK. Pseudomonas maltophilia bacteremia in children undergoing open heart sugery. JAMA 1981; 246: 1571-1574.

35. Flaherty JP, García-Houchins S, Chudy R, Arnow PM. An outbreak of gram-negative bacteremia traced to contaminated O-rings in reprocessed dialyzers. Ann Intern Med 1993; 119: 1072-1078.

36. Wishart MM, Riley TV. Infection with Pseudomonas maltophilia: hospital outbreak due contaminated disinfectant. Med J Aust 1976; 2: 710712.

37. Villarino ME, Stevens LE, Schable B, Mayers G, Miller JM, Burke JP, Jarvis WR. Risk factors for epidemic Xanthomonas maltophilia infection/ colonisation in intensive care unit patients. Infect Control Hosp Epidemiol 1992; 13: 201-206.

38. Public Health Laboratory Service. Ice as source of infection acquired in hospital. CDR Weekly 1993; 3: 53.

39. Fabe C, Rodríguez P, Cony-Malchoul P, Parneix P, Bebear C, Mougein J. Typage moleculaire par electrophorese en champ pulse de souches de Stenotrophomonas maltophilia and Pseudomonas cepacia. Curr Top Infect Dis Clin Microbiol 1996; 116: 52-83.

40. Campos-Herrero MI, Pena MJ, Pérez MC, Bordes A, Mosquera M Bacteriemia por Stenotrophomonas maltophilia. Enferm Infecc Microbiol Clin 1997; 15: 223-225.

41. Elting LS, Bodey GP. Septicaemia due to Xanthomonas species and non-aeruginosa species: increasing incidence of catheter-related infections. Medicine 1990; 69: 296-306.

42. Elting LS, Khardori N, Bodey GP, Fainstein V. Nosocomial infection caused by xanthomonas maltophilia: a case-control study of predispo- sing factors. Infect Control Hosp Epidemiol 1990; 11: 134-138.

43. Khardori N, Elting L, Wong E, Schable B, Bodey GP. Nosocomial infections due to Xanthomonas maltophilia ( Pseudomonas maltophilia ) in patients with cancer. Rev Infect Dis 1991; 12: 997-1003.

44. Muder RR, Harris AP, Muller S, Edmond M, Chow JW, Papadakis, K Wagener MW, Bodey GP Steckelberg JM. Bacteremia due to Stenetrophomonas ( Xanthomonas ) maltophilia: a prospective multi-center study of 91 episodes. Clin Infect Dis 1996; 22: 508-512.

45. Paton R, Miles RS, Amyes SGB. Biochemical properties of inducible blactamases produced from Xanthomonas maltophilia. Antimicrob Agents Chemother 1994; 38: 2143-2149.

46. Elkhaili H, Pompei D, Linger L, Kamili N, Monteil H, Jehl F. Cinetique de bactericidie de cefepime et cefpirome seuls ou associés a la gentamicine, amikacine ou ciprofloxacine sur Acinetobacter baumanii, Stenotrophomonas maltophilia et Enterobacter cloacae hyperproducteur de cephalosporinase. Pathol Biol 1996 ; 44: 367-373.

47. Fang GC, Madinger NE. Resistant nosocomial gram-negative bacillary pathogens: Acinetobacter baumanii, Xanthomonas maltophilia and Pseudomas cepacia. Curr Top Infect Dis Clin Microbiol 1996 ; 116 :5283.

48. Hohl P, Frei R, Aubry P. In vitro susceptibility of 33 clinical case isolates of Xanthomonas maltophilia: inconsistent correlation of agar dilution and of disk diffusion test results. Diagn Microbiol Infect Dis 1991; 14: 447-450.

49. Vartivarian S, Anaissie E, Bodey G, Sprigg H, Rolston K. A changing pattern of susceptibility of Xanthomonas maltophilia to antimicrobial agents: implications for therapy. Antimicrob Agents Chemother 1994; 38: 624-627.

50. Vartivarian SE, Papadakis KA, Palacios, JA Manning JT, Anaissie EJ. Mucocutaneous and soft tissue infections caused by Xanthomonas maltophilia: a new spectrum. Arch Intern Med 1994; 121: 969-973. 